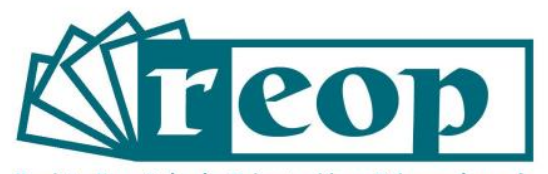

Revista Española de Orientación y Psicopedagogía

\title{
CYBERBULLING EN UNA MUESTRA UNIVERSITARIA ESPAÑOLA
}

\section{CYBERBULLYING IN A SPANISH UNIVERSITY SAMPLE}

\author{
Cristina Serrano García ${ }^{1}$ \\ Centro Universitario Cardenal Cisneros. Departamento de Educación y Psicología. Alcalá de \\ Henares, Madrid \\ Pilar Royo García
}

Centro Universitario Cardenal Cisneros. Departamento de Educación y Psicología. Alcalá de Henares, Madrid

\section{Cristina Laorden Gutiérrez}

Centro Universitario Cardenal Cisneros. Departamento de Educación y Psicología. Alcalá de Henares, Madrid

\section{Montserrat Giménez Hernández}

Centro Universitario Cardenal Cisneros. Departamento de Educación y Psicología. Alcalá de Henares, Madrid

\section{RESUMEN}

El estudio del cyberbulling se ha centrado en las etapas educativas previas a la universitaria, sobre todo en educación secundaria. El objetivo del trabajo es aportar datos sobre la prevalencia y las formas más frecuentes de cyberbulling en el contexto universitario. La muestra está formada por 776 estudiantes de 4 titulaciones de Grado (Magisterio en Educación Infantil, Magisterio en Educación Primaria, Educación Social y Psicología), matriculados de $1^{\circ}$ a $4^{\circ}$ curso (131 varones y 654 mujeres). Se utilizó el cuestionario ECIP-Q, de Del Rey et al. (2015), adaptado a un formato online, que identifica los roles de víctima, agresor, agresor victimizado y no implicado. Se utilizó una metodología cuantitativa. Los resultados muestran que el $4 \%$ de los participantes se identifica

${ }^{1}$ Correspondencia: Cristina Serrano García. Correo-e: cristina.serrano@cardenalcisneros.es 
como víctima; el 7,9\% como agresor y el 1,4\% como agresor-victimizado. Los roles de implicación se encuentran en los cuatro cursos, siendo mayor el porcentaje de víctimas y de agresores en el último curso. Las formas más frecuentes de victimización son: ser insultado a través del móvil o internet y ser excluido o ignorado de una red social, seguidas, a cierta distancia de las amenazas, la difusión de rumores o colgar información personal. Se concluye que el cyberbulling está presente en la universidad con todos los roles y que es necesario que los servicios de orientación universitarios implementen programas de intervención para prevenir y reducir el cyberbulling y potenciar buenas prácticas en las redes sociales, ya que el ciberacoso no parece remitir con la mayor madurez de los jóvenes.

Palabras clave: orientación, universidad, cyberbulling, víctimas, agresores.

\section{ABSTRACT}

The study of cyberbullying has focused on the educational stages prior to university, especially in Compulsory Secondary Education. The aim of this work is to provide data on the prevalence and the most frequent forms of cyberbullying in the university context. The sample is made up of 776 students from 4 Bachelor's degrees (Early Childhood Education, Primary Education, Social Education, and Psychology), enrolled in grades 1 to 4 (131 men and 654 women). The ECIP-Q questionnaire by Del Rey et al. (2015) was administered, adapted to an online format, which identifies the roles of victim, aggressor/offender, victimized aggressor and not involved. A quantitative methodology was used. Results: $4 \%$ of the participants identify themselves as victims; $7.9 \%$ as aggressor and $1.4 \%$ as aggressor victimized. Roles are distributed in the four courses and the percentage of victims and offenders is significantly higher in the last year. The most frequent forms of victimization are being insulted through the mobile phone or the Internet and being excluded or ignored from a social network, followed by threats, spreading rumors, or posting personal information. To conclude, cyberbullying is present in university and it is necessary for university guidance services to implement intervention programs to prevent and reduce cyberbullying and promote good practices in social networks, since cyberbullying does not seem to remit with the greater maturity of young people.

Key Words: guidance, higher education cyberbullying, victims, aggressors.

\section{Cómo citar este artículo:}

Serrano García, C., Royo-García, P., Laorden Gutiérrez, C. y Giménez Hernández, M., (2021). Cyberbulling en una muestra universitaria española. Revista Española de Orientación y Psicopedagogía, 32(2), 132-149. https://doi.org/10.5944/reop.vol.32.num.2.2021.31283

\section{Introducción}

En la última década, la comunidad educativa y científica ha mostrado un creciente interés por el cyberbulling, una nueva forma de violencia e intimidación entre iguales. Sin embargo, a pesar de 
la proliferación de estudios todavía se trata de un fenómeno muy complejo y difícil de definir y evaluar (Zych, et al, 2016).

En general, se entiende por cyberbulling el uso de la telefonía móvil, internet y demás medios telemáticos para ejercer acoso entre iguales (Tokunaga, 2010). Se considera que mantiene las principales características que definen el bullying: intencionalidad, reiteración, desequilibrio de poder y transgresión moral, aunque, además, tiene características propias, como la posibilidad de realizar un ataque en cualquier momento y lugar, el alcance de la exposición las 24 horas del día, el anonimato, la potencialidad de una mayor audiencia y la suplantación de identidad como una forma de causar grave daño moral (Del Rey et al., 2010; Gradinger, et al., 2010; Lucas, et al., 2016; Ortega-Ruiz, et al., 2016).

Las vías utilizadas en el cyberbulling son variadas, por ejemplo: mensajes ofensivos, groseros o amenazantes, acoso a través del móvil o de las redes sociales, difusión de rumores, grabaciones de agresiones físicas o vejaciones que son difundidas vía móvil o internet, difusión de fotos o vídeos comprometidos, revelación de información personal privada, exclusión de la comunicación en línea o suplantación de la identidad virtual, etc. (Garaigordobil, 2015; García Galera y Monferrer, 2009; Perren et al., 2012).

Aunque el número de estudios sobre el cyberbulling en España está aumentando en los últimos años a gran velocidad (Ortega-Ruiz y Zich, 2016), la mayoría de ellos se centra fundamentalmente en adolescentes, en la etapa educativa de Educación Secundaria Obligatoria (Domínguez, et al., 2017; Zych, et al., 2016;), y son muchos menos los estudios realizados en otras etapas educativas. Respecto a los trabajos llevados a cabo con estudiantes universitarios, casi todos se han realizado con muestras extranjeras y son pocos los que incluyen muestras españolas. Una excepción es el reciente estudio realizado en España que evidencia la aparición del acoso escolar en el contexto universitario, el cual determinó que existe relación entre haber sido víctima de ciberacoso, agente de ciberacoso y observador de ciberacoso (Méndez et al., 2019). También en el estudio de Dobarro, et al., (2017), con muestra española, encuentran la existencia de cyberbulling en estudiantes universitarios y una correlación entre cibervictimización y rendimiento académico, sentimiento de pertenencia y percepción de apoyo que el estudiante tiene.

Atendiendo a Prieto et al. (2015), el bullying y el cyberbulling no son fenómenos exclusivos de los niveles educativos precedentes e incluso adquieren matices específicos en el ámbito de la educación superior. Hay pocos estudios en esta etapa, pero se debe continuar investigando, para poder comparar estos resultados con otros trabajos sobre este tema (Dobarro et al., 2017).

Los roles identificados en las situaciones de bullying: víctima, agresor, observador y participante, también se encuentran cuando el acoso opera a través de la tecnología, de manera que se puede entender cómo se inician, mantienen, refuerzan y configuran las diferentes dinámicas en torno al acoso, y se ofrece pistas de una intervención que va más allá de atender a la víctima o sancionar al agresor (Salmivalli, 2010). Atendiendo a estos roles, Tokunaga (2010) apunta que aquellos estudiantes que no se sienten capaces de agredir a otros de forma presencial, pueden adoptar conductas de ciberacoso arropados por el anonimato que les ofrece la tecnología, reforzándose la idea de que esta modalidad de acoso se ha convertido en "un poderoso medio" para que los acosadores ejerzan su poder y control sobre las víctimas (Smith et al., citado de Cowie, 2013). Vergel et al. (2016) apuntan que la tecnología incrementa el riesgo de bullying y se constata que los agresores tienen menos empatía hacia sus víctimas que cuando el acoso se produce de forma presencial (Levy, citado de Smith y Yoon, 2012).

En cuanto a la prevalencia de cyberbulling en muestras universitarias, como se observa en la Tabla 1, los porcentajes de víctimas varían de menos del $1 \%$ en una muestra estona (Pörhöla et al., 2016) al 59,8\% detectado por Turan et al. (2011) con estudiantes turcos. En el caso de los agresores, los porcentajes se sitúan entre el 11\% (Brack y Caltabiano, 2014) y el 30\% (Prieto et al., 2015). 
Tabla 1

Prevalencia de cyberbulling en muestras universitarias extranjeras

\begin{tabular}{|c|c|c|}
\hline Autor/es & Muestra & Resultados \\
\hline Turan et al. (2011) & 579 estudiantes turcos & $\begin{array}{l}59,8 \% \text { han sufrido ciberacoso } \\
20,7 \% \text { a través de internet } \\
27,7 \% \text { a través de móvil } \\
51,7 \% \text { en ambos medios }\end{array}$ \\
\hline $\begin{array}{l}\text { Brack y Caltabiano } \\
(2014)\end{array}$ & $\begin{array}{l}164 \quad \text { estudiantes } \\
\text { australianos }\end{array}$ & $\begin{array}{l}62 \% \text { implicado en ciberacoso } \\
11 \% \text { agresores } \\
10 \% \text { víctimas } \\
17 \% \text { observadores }\end{array}$ \\
\hline Kokkinos et al. (2014) & $\begin{array}{ll}430 & \text { estudiantes } \\
\text { griegos } & \end{array}$ & $58,4 \%$ implicado en ciberacoso \\
\hline Prieto et al. (2015) & $\begin{array}{l}2493 \text { estudiantes } \\
\text { mexicanos }\end{array}$ & $\begin{array}{l}\text { CiberVíctimas: entre el } 20-40 \% \\
\text { CiberAgresores: } 30 \%\end{array}$ \\
\hline Pörhöla et al. (2016) & $\begin{array}{l}\text { Estudio cross-cultural: } \\
\text { Argentina } \quad(\mathrm{N}=969) ; \\
\text { Estonia } \quad(\mathrm{N}=1053) ; \\
\text { Finlandia }(\mathrm{N}=4403) \text { y } \\
\text { US }(\mathrm{N}=2082) .\end{array}$ & $\begin{array}{l}\text { Víctimas: } \\
\text { Argentina } 25 \% \\
\text { US } 11 \% \\
\text { Finlandia } 5 \% \\
\text { Estonia } 2 \% \\
\text { Cibervíctimas } \\
\text { menos de } 1 \% \text { en Estonia a } 4-5 \% \text { en } \\
\text { Argentina y EE. UU. }\end{array}$ \\
\hline Vergel, et al (2016) & $\begin{array}{l}571 \text { estudiantes } \\
\text { colombianos }\end{array}$ & CiberVíctimas: $5,7 \%$ \\
\hline Dobarro et al. (2017) & $\begin{array}{l}181 \text { estudiantes } \\
\text { españoles }\end{array}$ & $\begin{array}{l}22,1 \% \text { víctima de acoso } \\
29,3 \% \text { Observadores }\end{array}$ \\
\hline Jenaro et al. (2017) & $\begin{array}{lr}1593 & \text { estudiantes } \\
\text { españoles y bolivianos }\end{array}$ & $\begin{array}{l}5,1 \% \text { víctimas } \\
19,3 \% \text { observadores }\end{array}$ \\
\hline Redondo et al. (2017) & $\begin{array}{l}639 \text { estudiantes } \\
\text { bolivianos }\end{array}$ & $\begin{array}{l}\text { Víctimas: } 27,5 \% \\
\text { agresores: } 26,7 \%\end{array}$ \\
\hline
\end{tabular}

Fuente: Elaboración propia

La diversidad de frecuencias puede atribuirse a las distintas metodologías e instrumentos utilizados para la recogida de estos datos, así como a las posibles diferencias entre las poblaciones estudiadas (Dobarro et al., 2017; Romero y Plata, 2015). En cualquier caso, los datos confirman la existencia de cyberbulling en la universidad.

En cuanto a las formas de cyberbulling, ser acosado verbal o por escrito (17,7\% en Dobarro, et al., 2017; entre el $20 \%$ y $40 \%$ en Prieto et al., 2015) y ser excluido de ciertas actividades (30\% en Prieto et al., 2015), son las conductas de acoso que suelen presentar las frecuencias más altas, encontrándose la suplantación de la identidad (10\% en Dobarro et al., 2017) como la menos frecuente. Atendiendo a la intensidad percibida de la cibervictimización, Redondo et al. (2017) encuentran que el $25,4 \%$ de los evaluados han sido acosados de forma moderada, mientras que $2,1 \%$ de forma severa y Dobarro et al. (2017) encuentran que, del $22,1 \%$ de la muestra que se confesaba ser víctima de acoso, el 1,1\% lo eran de forma severa.

El cyberbulling, al igual que el bullying, compromete gravemente la convivencia en los centros educativos. De hecho, hoy la convivencia es también «ciberconvivencia» (Del Rey y Ojeda, 2018). Este hecho nos exige replantearnos las claves a tener en cuenta para la prevención de la violencia y la mejora de la convivencia también en la universidad, y más sabiendo que las relaciones socioafectivas entre los estudiantes marcan el ajuste emocional y relacional del sujeto (Sánchez Lacasa y Cerezo, 2011); y que el cyberbulling en estudiantes universitarios puede afectar al abandono universitario (Dobarro et al., 2017). 
El servicio y los programas de orientación universitarios son un lugar idóneo para trabajar desde la búsqueda continua de una convivencia positiva en la educación superior. En este sentido, mejorar el clima socioafectivo, la comunicación y la resolución de conflictos repercute en la gestación y mantenimiento del acoso (Carpio et al., 2013; Nocito, 2017). Por otro lado, como indican Ortega y Zych (2016), para que la cyberconvivencia sea posible, es necesario educar a los estudiantes en el uso seguro de las tecnologías de información y comunicación, sobre todo en los principios morales y éticos en contra de la violencia. Así, los programas de orientación en la universidad pueden contribuir a la transmisión de una ética y buenas prácticas en las redes sociales entre los estudiantes (Luengo, 2019).

Aunque las universidades no suelen tratar el tema del acoso entre estudiantes, algunas incluyen el tema de la convivencia a través de los servicios de orientación dirigidos a toda la comunidad universitaria y especialmente a los estudiantes, como es el caso del SOP (Servicio de Orientación Personal) de la Universidad Ramón Llul (Hernando et al., 2014) y el plan de orientación para los estudiantes de nuevo ingreso en los grados de Magisterio de Educación Infantil y Primaria en la Universidad de Zaragoza (Lizalde et al., 2018).

La presente investigación tiene como objetivo recabar información sobre el cyberbulling en una muestra universitaria española. Concretamente se pretende analizar cómo se distribuyen los diferentes roles de implicación (víctima, agresor, agresor victimizado y no implicado), estudiar la relación de dichos roles con la percepción que tienen los estudiantes del ambiente social del aula, y conocer las formas más frecuentes de ciberagresión y de cibervictimización en la muestra analizada. Estos datos pueden arrojar luz para futuras investigaciones o implicaciones psicoeducativas en este ámbito.

\section{Método}

\section{Muestra}

El procedimiento de muestreo llevado a cabo es no probabilístico por conveniencia. La muestra está constituida por 776 estudiantes. El rango de edad varía entre los 17 y los 58 años, el 95\% de los estudiantes se sitúa entre los 17 y los 26 años, siendo la media de 21,06 años y la DT de 3,775 . De ellos, $131(16,9 \%)$ son varones y $654(83,1 \%)$ son mujeres. La distribución por curso es la siguiente: $260(33,5 \%)$ de primer curso, $203(26,2 \%)$ de segundo curso, $137(17,7 \%)$ de tercer curso y $176(22,7 \%)$ de cuarto curso. Los participantes estudian 4 titulaciones de Grado (Magisterio en Educación Infantil 279 (46\%), Magisterio en Educación Primaria 233 (30,1\%), Educación Social 74 (9,4\%) y Psicología 190 (24,5\%) en centros universitarios.

\section{Instrumentos}

Para evaluar la implicación en cyberbulling de los estudiantes se ha utilizado la validación española realizada por Ortega-Ruiz et al. (2016) del European Cyberbullying Intervention Project Questionnaire (ECIP-Q, Del Rey et al., 2015). Se seleccionó este cuestionario por haber demostrado ser un instrumento fiable y empíricamente contrastado y por las ventajas y fortalezas 
de su validación, tal y como puede observarse en Ortega-Ruiz et al. (2016). Consta de 22 ítems agrupados en dos dimensiones: cibervictimización y ciberagresión. Los ítems siguen una escala tipo Likert de cinco opciones de respuesta referidas a un intervalo de tiempo de los últimos dos meses: $0=$ no; 1=sí, una o dos veces; $2=s i ́$, una o dos veces al mes; 3=sí, alrededor de una vez a la semana; y $4=s i ́$, más de una vez a la semana. El cuestionario permite identificar los siguientes roles en cyberbulling: víctima, agresor, agresor victimizado y no implicado. La fiabilidad en la versión de validación de la escala es $\alpha=0,97$ para la dimensión cibervictimización y $\alpha=0,93$ para la dimensión ciberagresión.

Para calcular los roles de implicación en cyberbulling en el cuestionario EICP-Q se consideraron los criterios de frecuencia de participación y repetición, de acuerdo con los criterios de Del Rey et al. (2015). Las víctimas fueron identificadas cuando se obtuvieron puntuaciones mayores o iguales a 2 (una vez al mes) en cualquiera de los ítems de victimización y puntuaciones iguales o menores que 1 (una o dos veces) en todos los ítems de agresión. Los agresores fueron aquellos que marcaron 2 o más (una vez al mes) en algún ítem de agresión y 1 o 0 (una o dos veces o nunca) en todos los ítems de victimización. Los agresores victimizados puntuaron igual o mayor a dos (una vez al mes) en cualquier ítem de la dimensión agresión y victimización. Los no implicados fueron considerados aquellos que obtuvieron una puntuación menor o igual que 1 (una o dos veces) tanto en los ítems de agresión como en los de victimización.

Por otro lado, también se evaluó en cada estudiante su percepción del ambiente social del aula y su sensación de bienestar en ella, a través de cuatro ítems elaborados ad hoc con cuatro alternativas de respuesta (mucho, bastante, poco o nada). Concretamente los ítems son: "Me siento a gusto en esta clase", "Considero que tengo amigos en clase"; "Puedo intervenir en clase sin problemas", "Considero que hay buen ambiente en clase". La consistencia obtenida con los datos de este estudio mediante $\alpha$ de Cronbach fue de $\alpha=0,729$.

\section{Procedimiento}

El cuestionario se aplicó en el primer cuatrimestre del curso 2017-2018 a través de un enlace online, de forma voluntaria y con la autorización paterna en el caso de los alumnos/as de primer curso menores de edad (19 estudiantes no habían cumplido 18 años en el momento de la aplicación del cuestionario). Esta autorización se facilitó a los padres en formato papel, donde se explicaba el objetivo del estudio y el proceso de gestión de datos. A todos los estudiantes se les explicó que las repuestas eran anónimas, confidenciales, que se requería sinceridad y que debían centrarse en lo ocurrido en el contexto universitario (no con los amigos de fuera de la universidad) y durante los últimos dos meses.

La clasificación de los participantes según los distintos roles de implicación en cyberbulling se realizó a partir de los datos brutos a través de un programa escrito para $R$ ( $R$ coreteam, 2018). Posteriormente, los datos fueron analizados con el paquete estadístico SPSS 24. Se calcularon los porcentajes de implicación en los diferentes roles del cyberbulling y se analizaron las diferencias en la distribución de dichos roles en función del curso, del género y de la titulación, a través de la prueba de chi-cuadrado $(x 2)$ y con la prueba $z$ para la comparación de las proporciones. Por otro lado, se analizaron las diferencias de medias entre los roles del cyberbulling en la percepción del ambiente social del aula y la sensación de bienestar en ella aplicando ANOVA factorial, con Bonferroni para los contrastes posteriores con un nivel de significación de .05. Por último, se analizaron los porcentajes de las distintas formas de ciberagresión y de cibervictimización, para lo cual se agruparon las puntuaciones mayores o iguales a uno. 


\section{Resultados}

Los resultados indican que, en la muestra total, el rol de víctima tiene un porcentaje de implicación del $4 \%$ (31 estudiantes), el rol de agresor del 7,9\% (61 estudiantes) y el rol de agresor-victimizado, del 1,4\% (11 estudiantes) (ver Tabla 2).

La prueba de Chi cuadrado indica que la distribución de los roles en función del curso es estadísticamente diferente $(x 2=23,898, g l=9, s i g=, 004)$. En relación con las víctimas, se observa que el porcentaje de alumnos de $1^{\circ}, 2^{\circ}$ y $3^{\circ}$ implicados en este rol es estadísticamente próximo entre sí (2,7\%, $2 \%$ y 5,5\% respectivamente), siendo el de los de $1^{\circ}$ y $2^{\circ}$ significativamente inferior al de los estudiantes de $4^{\circ}(6,8 \%)$, por lo que hay más víctimas de cyberbulling en el último curso de la muestra que en cada uno de los cursos inferiores. Respecto a los agresores, los resultados indican que el porcentaje de implicación en el rol de agresor va aumentando desde el primer curso $(3,5 \%)$, pasando por segundo y tercer curso $(8,9 \%$ y $8,0 \%$ respectivamente), hasta cuarto $(13,1 \mathrm{y} \%)$, encontrando diferencias estadísticamente significativas entre $1^{\circ} \mathrm{y}$ todos los demás cursos. Por otro lado, la distribución del porcentaje de implicación en el rol de agresor victimizado es estadísticamente similar en todos los cursos, siendo este, además, el rol menos frecuente, como cabría esperar (ver Tabla 2).

\section{Tabla 2}

Distribución de los roles de cyberbulling en función del curso y en la muestra total

\begin{tabular}{|c|c|c|c|c|c|c|c|}
\hline & & & \multicolumn{4}{|c|}{ Curso } & \multirow[t]{2}{*}{ Total } \\
\hline & & & $1^{0}$ & $2^{\circ}$ & $3^{\circ}$ & $4^{\circ}$ & \\
\hline \multirow{8}{*}{$\begin{array}{l}\text { Roles } \\
\text { en } \\
\text { cyber- } \\
\text { bulling }\end{array}$} & \multirow[t]{2}{*}{ No Implicado } & Recuento & $240_{a}$ & $179_{a, b}$ & $115 b, c$ & $139 c$ & 673 \\
\hline & & $\begin{array}{l}\% \text { dentro de } \\
\text { Curso }\end{array}$ & $92,3 \%$ & $88,2 \%$ & $83,9 \%$ & $79,0 \%$ & $86,7 \%$ \\
\hline & \multirow[t]{2}{*}{ Víctima } & Recuento & $7 \mathrm{a}$ & $4 a$ & $8 a, b$ & $12 \mathrm{~b}$ & 31 \\
\hline & & $\begin{array}{l}\% \text { dentro de } \\
\text { Curso }\end{array}$ & $2,7 \%$ & $2,0 \%$ & $5,8 \%$ & $6,8 \%$ & $4,0 \%$ \\
\hline & \multirow[t]{2}{*}{ Agresor } & Recuento & $9 a$ & $18 b$ & $11 \mathrm{~b}$ & $23 b$ & 61 \\
\hline & & $\begin{array}{l}\text { \% dentro de } \\
\text { Curso }\end{array}$ & $3,5 \%$ & $8,9 \%$ & $8,0 \%$ & $13,1 \%$ & $7,9 \%$ \\
\hline & \multirow{2}{*}{$\begin{array}{l}\text { Agresor } \\
\text { Victimizado }\end{array}$} & Recuento & $4 a$ & $2 a$ & $3 a$ & $2 a$ & 11 \\
\hline & & $\begin{array}{l}\% \text { dentro de } \\
\text { Curso }\end{array}$ & $1,5 \%$ & $1,0 \%$ & $2,2 \%$ & $1,1 \%$ & $1,4 \%$ \\
\hline & \multirow[b]{2}{*}{ Total } & Recuento & 260 & 203 & 137 & 176 & 776 \\
\hline & & $\begin{array}{l}\text { \% dentro de } \\
\text { Curso }\end{array}$ & $100 \%$ & $100 \%$ & $100 \%$ & $100 \%$ & $100 \%$ \\
\hline
\end{tabular}

Cada letra del subíndice denota un subconjunto de Curso cuyas proporciones de columna no difieren de forma significativa entre sí en el nivel ,05.

Fuente: Elaboración propia

Respecto al género, los resultados indican que no existen diferencias significativas en la distribución de los roles del cyberbulling entre hombres y mujeres $(x 2=, 163, g l=3$, sig=,983), siendo los porcentajes de implicación de los roles similares entre las chicas y los chicos $(3,9 \%$ y 
$4,6 \%$ respectivamente para el rol de víctima, el 7,9\% y 7,6\% para el rol de agresor y el $1,4 \%$ y $1,5 \%$ para el de agresor victimizado). Se es consciente de que la muestra es desigual al tratarse de estudios universitarios en los que se matriculan más chicas que chicos, pero interesaba ver cómo se distribuyen los diferentes roles del cyberbulling y si están presentes tanto en varones como en mujeres.

Tampoco se encuentran diferencias significativas en la distribución de los roles del cyberbulling en función de la titulación de grado de la muestra $(x 2=7,989, g l=6$, sig=,535), encontrándose los roles de víctima y agresor en cualquiera de ellas, por lo que parece que es un fenómeno que no se vincula a la titulación elegida, al menos en esta muestra.

Por otro lado, el ANOVA muestra diferencias significativas en función de los roles de implicación en cyberbulling en la percepción del ambiente social del aula en los cuatro ítems evaluados (Tabla 3).

\section{Tabla 3}

Media, desviación típica, $F$ y Significación del ANOVA en la percepción del ambiente social del aula en función de los roles de implicación en cyberbulling

\begin{tabular}{|c|c|c|c|c|c|c|}
\hline & & $\mathbf{N}$ & Media & $\begin{array}{l}\text { Desviación } \\
\text { estándar }\end{array}$ & $\mathbf{F}$ & Sig. \\
\hline \multirow{5}{*}{$\begin{array}{l}\text { Me siento } \\
\text { a gusto en } \\
\text { esta clase }\end{array}$} & No Implicado & 673 & 2,34 & ,794 & \multirow[t]{4}{*}{7,703} & \multirow[t]{4}{*}{,000 } \\
\hline & Ciber-Víctima & 31 & 1,77 & 1,023 & & \\
\hline & Ciber-Agresor & 61 & 2,07 & ,964 & & \\
\hline & $\begin{array}{l}\text { Ciber-Agresor } \\
\text { Victimizado }\end{array}$ & 11 & 1,82 & ,982 & & \\
\hline & Total & 776 & 2,29 & ,831 & & \\
\hline \multirow{5}{*}{$\begin{array}{l}\text { Considero } \\
\text { que tengo } \\
\text { amigos en } \\
\text { clase }\end{array}$} & No Implicado & 673 & 1,50 & ,646 & \multirow[t]{4}{*}{3,930} & \multirow[t]{4}{*}{,008 } \\
\hline & Ciber-Víctima & 31 & 1,10 &, 597 & & \\
\hline & Ciber-Agresor & 61 & 1,49 & ,649 & & \\
\hline & $\begin{array}{l}\text { Ciber-Agresor } \\
\text { Victimizado }\end{array}$ & 11 & 1,36 & ,924 & & \\
\hline & Total & 776 & 1,48 & ,652 & & \\
\hline \multirow{5}{*}{$\begin{array}{l}\text { Puedo } \\
\text { intervenir } \\
\text { en clase } \\
\text { sin } \\
\text { problemas }\end{array}$} & No Implicado & 673 & 2,38 & ,773 & \multirow[t]{4}{*}{3,745} & \multirow[t]{4}{*}{011} \\
\hline & Ciber-Víctima & 31 & 2,03 & ,983 & & \\
\hline & Ciber-Agresor & 61 & 2,31 & 847 & & \\
\hline & $\begin{array}{l}\text { Ciber-Agresor } \\
\text { Victimizado }\end{array}$ & 11 & 1,82 & 1,079 & & \\
\hline & Total & 776 & 2,36 & ,797 & & \\
\hline \multirow{5}{*}{$\begin{array}{l}\text { Considero } \\
\text { que hay } \\
\text { buen } \\
\text { ambiente } \\
\text { en clase }\end{array}$} & No Implicado & 673 & 1,92 & ,901 & \multirow[t]{4}{*}{6,301} & \multirow[t]{4}{*}{,000 } \\
\hline & Ciber-Víctima & 31 & 1,52 & 1,092 & & \\
\hline & Ciber-Agresor & 61 & 1,57 & 1,087 & & \\
\hline & $\begin{array}{l}\text { Ciber-Agresor } \\
\text { Victimizado }\end{array}$ & 11 & 1,18 & 1,168 & & \\
\hline & Total & 776 & 1,87 & ,938 & & \\
\hline
\end{tabular}

Fuente: Elaboración propia

Como se observa en las Tablas 4 y 5 , los contrastes realizados indican que los estudiantes que tienen rol de víctima, en relación con los compañeros no implicados en cyberbulling: se 
sienten menos a gusto en el aula $(p=0,000)$, consideran que tienen menos amigos en clase $(p=0,001)$, tienen una peor percepción de la posibilidad de intervenir en el aula sin problemas $(p=0,016)$ y no consideran que hay buen ambiente en clase $(p=0,017)$. También piensan que tienen significativamente menos amigos en clase que los implicados en el rol de agresor $(p=0,006)$. Por su parte, los estudiantes implicados en el rol de agresor difieren significativamente de los compañeros no implicados en cyberbulling en dos de los ítems evaluados. Así, los agresores se sienten menos a gusto en clase $(p=0,012)$ y consideran que hay peor ambiente en ella que los estudiantes no implicados $(p=0,005)$. Sin embargo, consideran que tienen amigos en clase y que pueden intervenir en ella sin problemas, en igual medida que los estudiantes no implicados en cyberbulling. Por último, los estudiantes con el rol de agresor-victimizado se sienten significativamente menos a gusto en el aula $(p=0,036)$, creen que no pueden intervenir en clase sin problemas $(p=0,019)$ y piensan que hay peor ambiente en clase $(p=0,009)$ que los estudiantes no implicados en cyberbulling.

\section{Tabla 4}

Comparaciones múltiples entre los roles del cyberbulling en la percepción del ambiente social del aula

\begin{tabular}{|c|c|c|c|c|c|c|c|}
\hline \multirow[t]{2}{*}{$\begin{array}{l}\text { Variable } \\
\text { dependiente }\end{array}$} & \multirow[t]{2}{*}{$\begin{array}{l}\text { (I) Roles en } \\
\text { cyberbulling }\end{array}$} & \multirow[t]{2}{*}{$\begin{array}{l}\text { (J) Roles en } \\
\text { cyberbulling }\end{array}$} & \multirow{2}{*}{$\begin{array}{l}\text { Diferencia } \\
\text { de medias } \\
\text { (I-J) }\end{array}$} & \multirow[t]{2}{*}{$\begin{array}{l}\text { Error } \\
\text { típico }\end{array}$} & \multirow[t]{2}{*}{ Sig. } & \multicolumn{2}{|c|}{$\begin{array}{c}\text { Intervalo de } \\
\text { confianza al } 95 \%\end{array}$} \\
\hline & & & & & & $\begin{array}{l}\text { Límite } \\
\text { superior }\end{array}$ & $\begin{array}{l}\text { Límite } \\
\text { inferior }\end{array}$ \\
\hline \multirow{12}{*}{$\begin{array}{l}\text { Me siento a } \\
\text { gusto en } \\
\text { esta clase }\end{array}$} & \multirow[t]{3}{*}{ No Implicado } & Ciber-Víctima &, $568\left(^{*}\right)$ & 0,151 & 0,000 & 0,27 & 0,86 \\
\hline & & Ciber-Agresor &, $276\left(^{*}\right)$ & 0,110 & 0,012 & 0,06 & 0,49 \\
\hline & & $\begin{array}{l}\text { Ciber-Agresor } \\
\text { Victimizado }\end{array}$ &, $524\left(^{*}\right)$ & 0,250 & 0,036 & 0,03 & 1,01 \\
\hline & \multirow[t]{3}{*}{ Ciber-Víctima } & No Implicado &,$- 568\left(^{*}\right)$ & 0,151 & 0,000 & $-0,86$ & \\
\hline & & Ciber-Aaresor & $-0,291$ & 0.181 & 0.108 & -0.65 & $\begin{array}{l}0,27 \\
0,06\end{array}$ \\
\hline & & $\begin{array}{l}\text { Ciber-Agresor } \\
\text { Victimizado }\end{array}$ & $-0,044$ & 0,288 & 0,879 & $-0,61$ & 0,52 \\
\hline & \multirow[t]{3}{*}{ Ciber-Agresor } & No Implicado &,$- 276\left(^{*}\right)$ & 0,110 & 0,012 & $-0,49$ & 0,06 \\
\hline & & Ciber-Víctima & 0,291 & 0,181 & 0,108 & $-0,06$ & 0,65 \\
\hline & & $\begin{array}{l}\text { Ciber-Agresor } \\
\text { Victimizado }\end{array}$ & 0,247 & 0,269 & 0,358 & $-0,28$ & 0,78 \\
\hline & \multirow[t]{3}{*}{$\begin{array}{l}\text { Ciber-Agresor } \\
\text { Victimizado }\end{array}$} & No Implicado &,$- 524\left(^{*}\right)$ & 0,250 & 0,036 & $-1,01$ & 0,03 \\
\hline & & Ciber-Víctima & 0,044 & 0,288 & 0,879 & $-0,52$ & 0,61 \\
\hline & & Ciber-Agresor & $-0,247$ & 0,269 & 0,358 & $-0,78$ & 0,28 \\
\hline \multirow{6}{*}{$\begin{array}{l}\text { Considero } \\
\text { que tengo } \\
\text { amigos en } \\
\text { clase }\end{array}$} & \multirow[t]{3}{*}{ No Implicado } & Ciber-Víctima & ,402(*) & 0,119 & 0,001 & 0,17 & 0,64 \\
\hline & & Ciber-Agresor & 0,007 & 0,087 & 0,932 & $-0,16$ & 0,18 \\
\hline & & $\begin{array}{l}\text { Ciber-Agresor } \\
\text { Victimizado }\end{array}$ & 0,136 & 0,197 & 0,492 & $-0,25$ & 0,52 \\
\hline & \multirow[t]{3}{*}{ Ciber-Víctima } & No Implicado &,$- 402\left(^{*}\right)$ & 0,119 & 0,001 & $-0,64$ & $0,17^{-}$ \\
\hline & & Ciber-Agresor &,$- 395\left(^{*}\right)$ & 0,143 & 0,006 & $-0,68$ & 0,11 \\
\hline & & $\begin{array}{l}\text { Ciber-Agresor } \\
\text { Victimizado }\end{array}$ & $-0,267$ & 0,228 & 0,241 & $-0,71$ & 0,18 \\
\hline
\end{tabular}

*. La diferencia de medias es significativa al nivel .05 .

Fuente: Elaboración propia 


\section{Tabla 5}

Comparaciones múltiples entre los roles del cyberbulling en la percepción del ambiente social del aula (Continuación)

\begin{tabular}{|c|c|c|c|c|c|c|c|}
\hline \multirow[t]{2}{*}{$\begin{array}{l}\text { Variable } \\
\text { dependiente }\end{array}$} & \multirow[t]{2}{*}{$\begin{array}{l}\text { (I) Roles en } \\
\text { cyberbulling }\end{array}$} & \multirow[t]{2}{*}{$\begin{array}{l}\text { (J) Roles en } \\
\text { cyberbulling }\end{array}$} & \multirow{2}{*}{$\begin{array}{c}\text { Diferencia } \\
\text { de medias } \\
\text { (I-J) }\end{array}$} & \multirow[t]{2}{*}{$\begin{array}{l}\text { Error } \\
\text { típico }\end{array}$} & \multirow[t]{2}{*}{ Sig. } & \multicolumn{2}{|c|}{$\begin{array}{c}\text { Intervalo de } \\
\text { confianza al } 95 \%\end{array}$} \\
\hline & & & & & & $\begin{array}{l}\text { Límite } \\
\text { superior }\end{array}$ & $\begin{array}{l}\text { Límite } \\
\text { inferior }\end{array}$ \\
\hline \multirow{6}{*}{$\begin{array}{l}\text { Considero } \\
\text { que tengo } \\
\text { amigos en } \\
\text { clase }\end{array}$} & \multirow[t]{3}{*}{ Ciber-Agresor } & No Implicado & $-0,007$ & 0,087 & 0,932 & $-0,18$ & 0,16 \\
\hline & & Ciber-Víctima & ,395(*) & 0,143 & 0,006 & 0,11 & 0,68 \\
\hline & & $\begin{array}{l}\text { Ciber-Agresor } \\
\text { Victimizado }\end{array}$ & 0,128 & 0,212 & 0,547 & $-0,29$ & 0,55 \\
\hline & \multirow{3}{*}{$\begin{array}{l}\text { Ciber-Agresor } \\
\text { Victimizado }\end{array}$} & No Implicado & $-0,136$ & 0,197 & 0,492 & $-0,52$ & 0,25 \\
\hline & & Ciber-Víctima & 0,267 & 0,228 & 0,241 & $-0,18$ & 0,71 \\
\hline & & Ciber-Agresor & $-0,128$ & 0,212 & 0,547 & $-0,55$ & 0,29 \\
\hline \multirow{12}{*}{$\begin{array}{l}\text { Puedo } \\
\text { intervenir } \\
\text { en clase sin } \\
\text { problemas }\end{array}$} & \multirow[t]{3}{*}{ No Implicado } & Ciber-Víctima &, $351\left(^{*}\right)$ & 0,146 & 0,016 & 0,07 & 0,64 \\
\hline & & Ciber-Agresor & 0,072 & 0,106 & 0,498 & $-0,14$ & 0,28 \\
\hline & & $\begin{array}{l}\text { Ciber-Agresor } \\
\text { Victimizado }\end{array}$ &, $565\left(^{*}\right)$ & 0,241 & 0,019 & 0,09 & 1,04 \\
\hline & \multirow[t]{3}{*}{ Ciber-Víctima } & No Implicado &,$- 351\left(^{*}\right)$ & 0,146 & 0,016 & $-0,64$ & 0,07 \\
\hline & & Ciber-Agresor & $-0,279$ & 0,175 & 0,111 & $-0,62$ & 0,06 \\
\hline & & $\begin{array}{l}\text { Ciber-Agresor } \\
\text { Victimizado }\end{array}$ & 0,214 & 0,278 & 0,442 & $-0,33$ & 0,76 \\
\hline & \multirow{3}{*}{ Ciber-Agresor } & No Implicado & $-0,072$ & 0,106 & 0,498 & $-0,28$ & 0,14 \\
\hline & & Ciber-Víctima & 0,279 & 0,175 & 0,111 & $-0,06$ & 0,62 \\
\hline & & $\begin{array}{l}\text { Ciber-Agresor } \\
\text { Victimizado }\end{array}$ & 0,493 & 0,260 & 0,058 & $-0,02$ & 1,00 \\
\hline & \multirow[t]{3}{*}{$\begin{array}{l}\text { Ciber-Agresor } \\
\text { Victimizado }\end{array}$} & No Implicado &,$- 565\left(^{*}\right)$ & 0,241 & 0,019 & $-1,04$ & 0,09 \\
\hline & & Ciber-Víctima & $-0,214$ & 0,278 & 0,442 & $-0,76$ & 0,33 \\
\hline & & Ciber-Agresor & $-0,493$ & 0,260 & 0,058 & $-1,00$ & 0,02 \\
\hline \multirow{12}{*}{$\begin{array}{l}\text { Considero } \\
\text { que hay } \\
\text { buen } \\
\text { ambiente en } \\
\text { clase }\end{array}$} & \multirow[t]{3}{*}{ No Implicado } & Ciber-Víctima &, $407\left(^{*}\right)$ & 0,171 & 0,017 & 0,07 & 0,74 \\
\hline & & Ciber-Agresor &, $349\left(^{*}\right)$ & 0,124 & 0,005 & 0,11 & 0,59 \\
\hline & & $\begin{array}{l}\text { Ciber-Agresor } \\
\text { Victimizado }\end{array}$ &, $741\left(^{*}\right)$ & 0,282 & 0,009 & 0,19 & 1,30 \\
\hline & \multirow[t]{3}{*}{ Ciber-Víctima } & No Implicado &,$- 407\left(^{*}\right)$ & 0,171 & 0,017 & $-0,74$ & 0,07 \\
\hline & & Ciber-Agresor & $-0,058$ & 0,205 & 0,779 & $-0,46$ & 0,34 \\
\hline & & $\begin{array}{l}\text { Ciber-Agresor } \\
\text { Victimizado }\end{array}$ & 0,334 & 0,326 & 0,306 & $-0,31$ & 0,97 \\
\hline & \multirow[t]{3}{*}{ Ciber-Agresor } & No Implicado &,$- 349\left(^{*}\right)$ & 0,124 & 0,005 & $-0,59$ & 0,11 \\
\hline & & Ciber-Víctima & 0,058 & 0,205 & 0,779 & $-0,34$ & 0,46 \\
\hline & & $\begin{array}{l}\text { Ciber-Agresor } \\
\text { Victimizado }\end{array}$ & 0,392 & 0,304 & 0,198 & $-0,21$ & 0,99 \\
\hline & \multirow[t]{3}{*}{$\begin{array}{l}\text { Ciber-Agresor } \\
\text { Victimizado }\end{array}$} & No Implicado &,$- 741\left(^{*}\right)$ & 0,282 & 0,009 & $-1,30$ & 0,19 \\
\hline & & Ciber-Víctima & $-0,334$ & 0,326 & 0,306 & $-0,97$ & 0,31 \\
\hline & & Ciber-Agresor & $-0,392$ & 0,304 & 0,198 & $-0,99$ & 0,21 \\
\hline
\end{tabular}

*. La diferencia de medias es significativa al nivel .05.

Fuente: Elaboración propia 
Respecto al modo en que se lleva a cabo el cyberbulling, los resultados indican que las formas más frecuentes de agresión en los estudiantes universitarios de la muestra son fundamentalmente decir palabras malsonantes (insultar), directamente o a través de terceras personas, usando el móvil o internet, y excluir a alguien de una red social. Seguido a bastante distancia se encuentra crear una cuenta falsa para hacerse pasar por otra persona, retocar fotos de alguien colgadas en internet, amenazar y difundir rumores (ver Figura 1).

\section{Figura 1}

\section{Formas de agresión en cyberbulling}

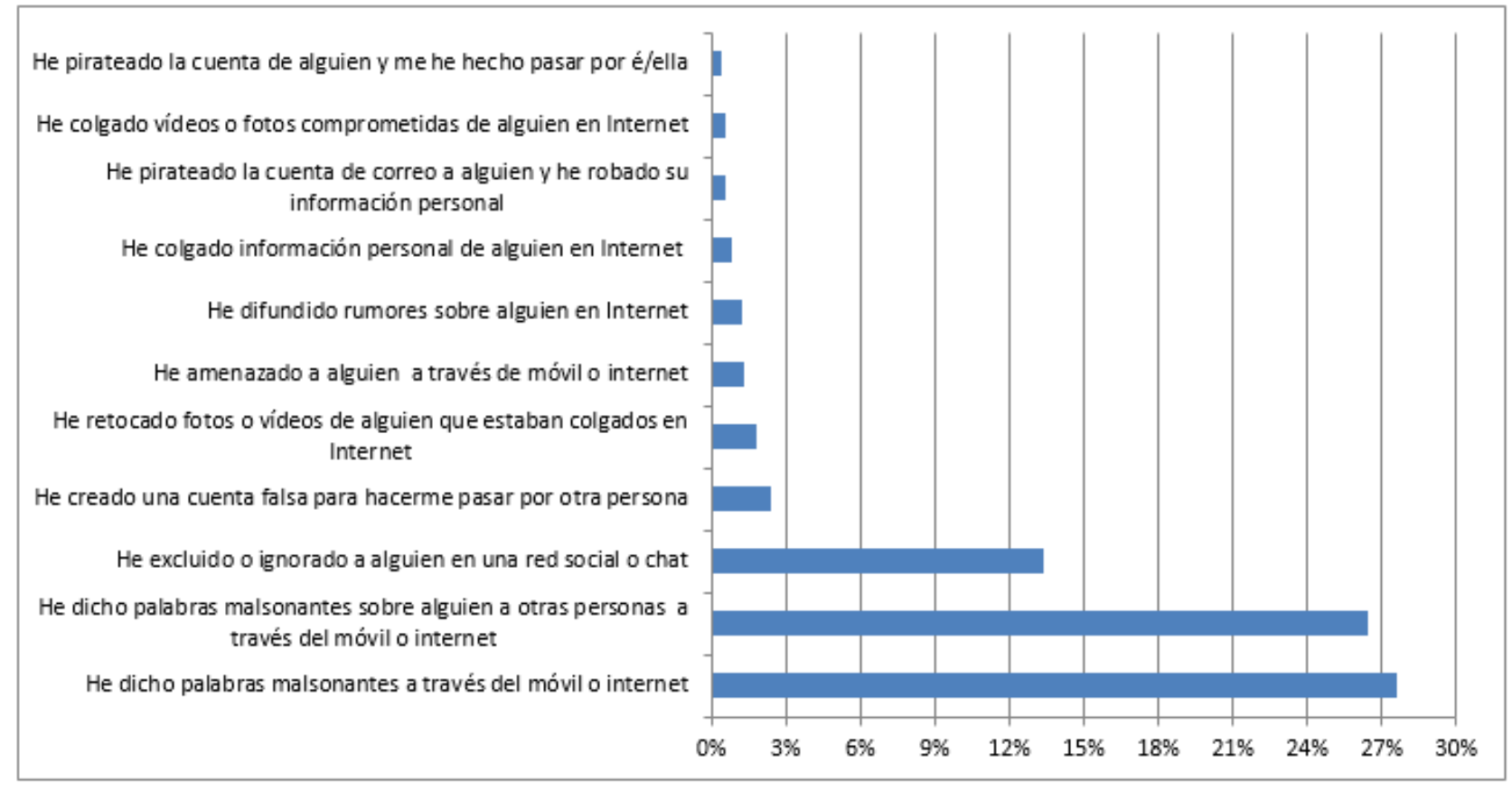

Fuente: Elaboración propia

A su vez, las formas más frecuentes de victimización en los estudiantes universitarios son: ser insultado utilizando el móvil o internet a través de terceras personas o directamente y ser excluido o ignorado de una red social, seguido a cierta distancia de ser amenazado, difundir rumores sobre él y haber colgado información personal en el móvil o internet (ver Figura 2). 


\section{Figura 2}

Formas de victimización en cyberbulling

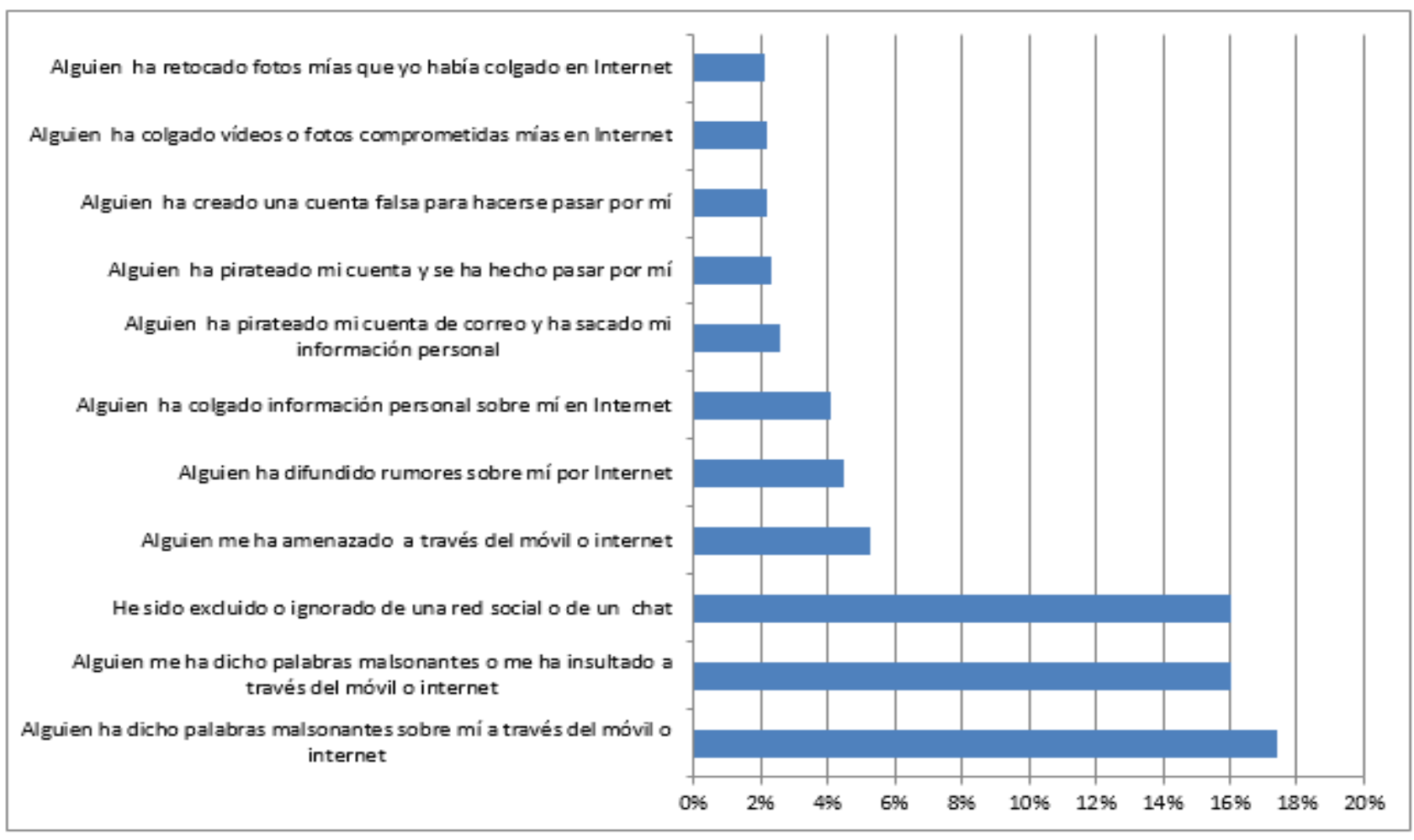

Fuente: Elaboración propia

\section{Conclusiones y Discusión}

Los resultados anteriormente presentados nos indican la existencia de cyberbulling en las aulas universitarias estudiadas en la línea de lo encontrado en investigaciones anteriores (Prieto et al., 2015; Dobarro et al., 2017), y la presencia de todos los roles o perfiles de cyberbulling analizados: víctima, agresor, agresor-victimizado y no implicado.

Los roles estudiados están presentes en todos los cursos de los grados de la muestra analizada. Además, los datos permiten, en términos generales, afirmar que hay más conductas de cyberbulling de agresores y de víctimas en el último curso. Cabría esperar que la mayor madurez de los alumnos del último curso y sus expectativas profesionales más cercanas los llevaran a no hacer un uso inadecuado de las redes sociales, pero es el curso en el que más casos encontramos tanto de víctimas $(6,8 \%)$ como de agresores $(13,1 \%)$, lo que podría deberse entre otras cosas al mayor acceso y manejo de las tecnologías con la edad. El hecho de encontrar un mayor porcentaje de cyberbulling en los estudiantes de $4^{0}$ va en la línea señalada por Garaigordobil (2015) que indica que no hay disminución con la edad porque son conductas que se mantienen en el tiempo o incluso pueden aumentar y que ponen de manifiesto la corriente actual de normalizar algunas conductas agresivas tal y como señalan Fernández et al. (2011). También van en la línea de los pocos estudios realizados sobre el cyberbulling en la universidad, encontrando que lo sufren más los estudiantes de mayor edad (Dorantes, 2016). 
En el presente estudio se ha encontrado un mayor número de universitarios en el rol de agresor que en los roles de víctimas y de agresor-victimizado, aunque estudios previos encuentran porcentajes muy similares al comparar los diferentes roles (Brack y Caltabiano, 2014; Redondo et al., 2017 y Prieto et al., 2015).

Analizando la muestra total, se observa que los datos encontrados se enmarcan entre los de otras investigaciones con muestras universitarias extranjeras. Más concretamente, los porcentajes encontrados de víctimas en el presente trabajo (4\%) se acercan sobre todo a los encontrados en la muestra de estudiantes argentinos del estudio transcultural de Pörhöla et al. (2016), que encuentran entre un 4-5\% de cibervíctimas. Respecto a los agresores, se encuentran por debajo de los escasos estudios que contemplan este rol en universitarios. El 7,9\% en la muestra total se sitúa por debajo de la horquilla del $11 \%$ al $30 \%$ de los estudios de Brack y Caltabiano (2014) y Prieto et al. (2015) con muestras de estudiantes australianos y mexicanos respectivamente. Estas diferencias en el porcentaje de ciberagresores pueden deberse, por ejemplo, al instrumento de medida utilizado o la normalización de las conductas de los agresores que les impide identificarse como tales.

Los resultados presentados en este estudio con respecto a las diferencias de género no muestran diferencias significativas entre varones y mujeres, aunque aparecen más cibervíctimas entre los varones en la muestra utilizada, lo que podría justificarse por el número muy superior de mujeres en las titulaciones estudiadas y en los datos de otras investigaciones, como la Fundación ANAR (2016), que apuntan que el acoso aumenta en mujeres cuando se trata de cyberbulling.

En la misma línea, las diferencias entre las titulaciones de grado estudiadas (Magisterio de Educación Primaria, Magisterio de Educación Infantil, Educación Social y Psicología) no son significativas. Sería interesante analizar titulaciones de otras áreas de conocimiento, ya que hay estudios con estudiantes universitarios mexicanos que encuentran un mayor porcentaje de cyberbulling en titulaciones del área técnica que del área de humanidades (Dorantes, 2016). Llama especialmente la atención que, en las titulaciones analizadas, que son de carácter social, encontremos un porcentaje relevante de ciberacoso. Los estudiantes que se han identificado como agresores no han renunciado a la deseabilidad social al reconocerse como tales. Esto, junto al hecho de que en los ítems asociados a la agresión se recogen conductas concretas, pero nunca se alude al concepto de acoso explícitamente, hace pensar que posiblemente los "agresores" estén naturalizando unas conductas de poco respeto a los iguales. En otras palabras, posiblemente estas personas no se reconozcan a sí mismas como agresoras, y se hayan habituado a relacionarse con los iguales de esta manera. En este sentido, se considera necesario introducir en los centros universitarios planes de convivencia basados en un diálogo permanente y una reflexión compartida en la relación con los otros. Desde los servicios de orientación se debe facilitar la creación de un clima adecuado para favorecer el aprendizaje, pero también las relaciones interpersonales y la resolución de conflictos. Sería interesante comparar los resultados de la aplicación del Ciberprogram 2.0 (Garaigordobil y Martínez-Valderrey, 2014) con muestras universitarias, centrándose en la empatía entre compañeros y el campo emocional de los conflictos

Así, por ejemplo, en las conductas de agresión destacan el insulto o decir palabras malsonantes, entre iguales o a terceras personas, así como excluir o ignorar a alguien a través del móvil o internet, resultados que podrían compararse con las formas de cyberbulling más frecuentes detectadas en estudios anteriores, donde ser acosado verbalmente o por escrito (Dobarro et al., 2017 y Prieto et al., 2015) y ser excluido de ciertas actividades (Prieto et al., 2015), son las más comunes. Estas conductas coinciden con las señaladas por las personas en el rol de víctimas, como las que más reciben. Sería interesante analizar la percepción de los propios agresores sobre estas conductas y su reflexión acerca de su nivel de transgresión hacia normas de respeto hacia los demás o niveles de violencia hacia los otros. Conviene destacar que los agresores, que se perciben sin problemas para intervenir en clase y con más amigos que las 
víctimas, manifiestan sentirse menos a gusto en clase que los no implicados. Se considera que puede facilitar la relación en las aulas la presencia de normas de convivencia claramente visibles para los estudiantes que traten el respeto, el uso de dispositivos electrónicos, uso de grabaciones y realización de fotografías sin permisos adecuados, etc.

Por otro lado, como indican Ortega y Zych (2016), para que la ciberconvivencia sea posible, es necesario educar a los estudiantes de todos los niveles educativos en el uso seguro de las tecnologías de información y comunicación, sobre todo en los principios morales y éticos en contra de la violencia.

Aunque el alcance de la muestra solo implica a titulaciones universitarias relacionadas con la educación (magisterio, educación social y psicología) se piensa que estudiar el cyberbulling en ellas es muy relevante, ya que estos universitarios en su futuro profesional tienen que estar preparados para contribuir a la transmisión de un código ético y buenas prácticas en las redes sociales en sus propios alumnos o educandos (Avilés, 2013; Luengo, 2013), bien directamente como profesores/educadores o a través de la elaboración de programas de intervención psicológica, por lo que su análisis puede ayudar a determinar necesidades en la formación inicial de estos estudiantes para su desempeño profesional futuro. Lo mismo ocurre con la formación específica sobre temas de convivencia y resolución de conflictos para los docentes, por lo que desde la orientación en los centros se trasmiten necesidades en este sentido. En futuras investigaciones sería necesario aplicar algunas de las técnicas prácticas señaladas por Tresgallo (2020) y obtener conclusiones sobre su uso en la etapa universitaria. Esto podría facilitar la labor de prevención en los servicios de orientación universitarios.

Se considera que, en todas las etapas educativas, los alumnos tienen derecho a aprender en un ambiente seguro y vinculado a emociones positivas, incluso en la universidad. Sin embargo, los estudiantes víctimas de cyberbulling parecen tener una peor percepción del clima de la clase y de su bienestar en ella. Concretamente, se sienten menos a gusto en las aulas, se perciben con menos amigos y consideran que pueden intervenir menos en el desarrollo de las clases.

Por otro lado, sería interesante analizar las características sociales de los estudiantes implicados en cyberbulling y la aceptación social dentro del grupo, ya que cabe esperar un bajo nivel de apoyo social relacionado con la cibervictimización y con la ciberagresión (Romera et al., 2016) o, como encuentran Sánchez Lacasa y Cerezo (2011) en etapas más tempranas, los agresores, a pesar de ser rechazados, son valorados como fuertes y con cierta popularidad, lo que les sitúa en una posición de mayor poder frente a las víctimas, quienes son consideradas cobardes.

En conclusión, a pesar de las limitaciones de la muestra, se piensa que los resultados de este estudio son de utilidad para favorecer la reflexión de los responsables de las instituciones universitarias, de sus claustros y de sus servicios de orientación. Parece evidente que es imprescindible educar en la "ética tecnológica", en el uso adecuado de todo lo que esta nos aporta (Avilés, 2013; Luengo, 2014) e incorporarla a las normativas de funcionamiento de los centros. Los estudiantes de esta etapa podrían ser más hábiles en el uso de dispositivos electrónicos, lo que podría llevarlos a realizar ataques más sofisticados o, por el contrario, a tener una mayor seguridad online o más habilidades para bloquearlos (Zych et al., 2016). Son chicos y chicas acostumbrados a recibir información rápidamente, a estar conectados, a hablar con varias personas a la vez de temas diferentes, a procesar en paralelo (Aguilar, 2011). Los medios de comunicación e Internet se convierten en medios trascendentales en su desarrollo y en la construcción de su identidad (González Calatayud, 2016). Pero el hecho de que sean nativos digitales no significa que sepan hacer un uso adecuado de la red conforme a lo que establece la ley, es por ello por lo que los casos de conductas delictivas en Internet estén aumentando de forma importante en los últimos tiempos (Tovar et al., 2016). 
Además, la existencia de violencia en las aulas, en cualquier nivel educativo, debe hacer reflexionar sobre la naturalización de la violencia, las diferentes transgresiones en la convivencia de cualquier centro educativo y los caminos adecuados para alcanzar la convivencia positiva (Fernández et al., 2011). En este sentido sería recomendable tener planes para mejora de la convivencia destinados a todos los universitarios y no solo a los de nuevo ingreso puesto que según marcan los datos presentados, los problemas de acoso aparecen en todos los cursos.

Las universidades pueden y deben ser un lugar donde se produzcan cambios educativos en los modos de relacionarse con los demás y de alcanzar una convivencia positiva que pueda verse reflejada en los contextos futuros, profesionales y personales, de los estudiantes universitarios. Para ello hay que tener en cuenta los datos que proporciona la investigación y los programas de formación que permitan dotar de herramientas preventivas que reduzcan estos conflictos o se aprenda a resolverlos.

Las futuras investigaciones deben orientarse a profundizar en las características del cyberbulling y sus consecuencias en la educación superior, analizar si tiene aspectos diferenciales respecto a etapas educativas anteriores y diseñar programas de intervención para prevenir y reducir el cyberbulling en estas edades.

\section{Referencias bibliográficas}

Aguilar, M.V. (2011). Jóvenes en la sociedad red: nuevas habilidades para las nuevas generaciones. En Martínez, F. y Solano, I. M. (Eds.), Comunicación y relaciones sociales de los jóvenes en la red (pp. 97-109). Marfil.

Avilés, J.M. (2013). Análisis psicosocial del cyberbulling: claves para una educación moral. Papeles del Psicólogo, 34 (1), 65-73. https://www.redalyc.org/pdf/778/77825706007.pdf

Brack, K. y Caltabiano, N. (2014). Cyberbullying and self-esteem in Australian adults. Cyberpsychology: Journal of Psychosocial Research on Cyberspace, 8 (2), artícle 7. https://doi.org/10.5817/CP2014-2-7, https://cyberpsychology.eu/article/view/4305/3354

Carpio, C., Tejero, J.M. y García Díaz, V. (2013) Análisis de los factores influyentes en un ambiente escolar con violencia. Revista de Orientación y Psicopedagogía, 24(3), 124 134. https://doi.org/10.5944/reop.vol.24.num.3.2013.11249

Cowie, H.A. (2013) Cyberbullying and its impact on young people's emotional health and wellbeing The Psychiatrist, 37, 167-170. https://doi.org/10.1192/pb.bp.112.040840

Del Rey, R., Casas, J.A., Ortega-Ruiz, R., Schultze-Krumbholz, A., Scheithauer, H., Smith, P.,Thompson, F., Barkoukis, V., Tsorbatzoudis, H., Brighi, A., Guarini, A., Pyzalski, J. y Plichta, P. (2015). Structural validation and cross-cultural robustness of the European Cyberbullying Intervention Project Questionnaire. Computers in Human Behavior, 50, 141147.https://doi.org/10.1016/j.chb.2015.03.065.

Del Rey, R., Flores, J., Garmendia, M., Martínez, G., Ortega, R. y Tejerina, O. (2010). Protocolo de actuación escolar ante el cyberbulling. EMICI. Equipo Multidisciplinar de Investigación sobre Cyberbulling. Junta de Andalucía. 
Del Rey, R. y Ojeda, M, (2018). Claves para prevenir el acoso y el ciberacoso. Participación Educativa. Revista del Consejo Escolar del Estado, 5 (8), 131-141. https://doi.org/10.4438/1886-5097

Dobarro, A., Carbajal, R., Ayala, I., Herrero, J. y Bernardo, A. (2017). Cyberbulling y violencia en la universidad, ¿cómo afecta al abandono? Revista de Estudios e Investigación en Psicología y Educación, Extr. 14, 182-183. https://doi.org/10.17979/reipe.2017.0.14.2737

Dorantes, J. J. (2016) Redes sociales y el cyberbullying en la Universidad Veracruzana. Revista Ensayos Pedagógicos Edición Especial, 169-188. https://doi.org/10.15359/rep.esp-16.9

Domínguez, J., Álvarez, E. y Vázquez, E. (2017). Dimensiones predictivas del constructo de violencia escolar en educación secundaria obligatoria. Revista de Investigación educativa 35(2), 337-351.https://dx.doi.org/10.6018//rie.35.2.259471

Hernando, N., Vilaregut Puigdesens, A., Palma Sevillano, C., Dotras Ruscalleda, P., Llinares-Fité, M. y Dalmau-Jordá, C. (2014). Estudio descriptivo de un modelo de orientación universitario. Revista de Orientación y Psicopedagogía, 25 (3), 106-120. https://doi.org/10.5944/reop.vol.25.num.3.2014.13861

Fernández, I., Pericado, F.J. y Andrés, M. (2011). Reflexiones en torno al concepto de violencia en las aulas. Revista Pulso, 34, 195-210.

https://revistas.cardenalcisneros.es/index.php/PULSO/article/view/120/96

Fundación Anar y Mutua Madrileña (2016). II estudio sobre acoso escolar y cyberbulling según los afectados.https://www.anar.org/wp-content/uploads/2017/04/INFORME-II-ESTUDIOCYBERBULLING.pdf

Garaigordobil, M y Martínez-Valderrey, V. (2014). Cyberprogram 2.0. Programa de intervención para prevenir y reducir el ciberbullying. Pirámide.

Garaigordobil, M. (2015). Cyberbulling en adolescentes y jóvenes del País Vasco: Cambios con la edad. Anales de psicología, 31(3), 1069-1076.https://doi.org/10.6018/analesps.31.3.179151

García Galera, M.C. y Monferrer, J.M. (2009). Propuesta de análisis teórico sobre el uso del teléfono móvil en adolescentes. Comunicar. Revista Científica de Educomunicación, 33, 83-92. https://doi.org/10.3916/c33-2009-02-008

Gradinger, P., Strohmeier, D. y Spiel, C. (2010). Definition and Measurement of cyberbulling. Cyberpsychology: Journal of Psychosocial Research on Cyberspace, 1, 114. https://cyberpsychology.eu/article/view/4235/3280

González Calatayud, V. (2016). Prevalencia del ciberacoso en las aulas de educación secundaria obligatoria y la percepción del profesorado. ]Tesis Doctoral, Universidad de Murcia].

Jenaro, C.; Flores, N. y Frías, C.P. (2017). Anxiety and Depression in Cyberbullied College Students: A Retrospective Study. Journal of Interpersonal Violence, 2021, 36 (1-2), 579602. https://doi.org/10.1177/0886260517730030

Kokkinos, C. M., Antoniadou, N. y Markos, A. (2014). Cyber-bullying: An investigation of the psychological profile of university student participants. Journal of Applied Developmental Psychology, 35(3), 204-214. https://doi.org/10.1016/j.appdev.2014.04.001

Lizalde Gil, M., Casanova López, O., Serrano Pastor, R. M. ㄹ. y Escolano Pérez, E. (2018). Plan de orientación universitaria para los estudiantes de nuevo ingreso. Programación de acciones y elaboración de materiales. Revista de orientación y psicopedagogía, 29(2), 41-54. https://doi.org/10.5944/reop.vol.29.num.2.2018.23152 
Lucas, B., Pérez, A. y Giménez, M. (2016). La evaluación del cyberbulling: Situación actual y retos futuros. Papeles del Psicólogo, 37(1), 27-35.

http://www.papelesdelpsicologo.es/pdf/2659.pdf

Luengo, J. A. (2013). Promover valores y ética en las relaciones digitales: la necesidad de actuar cuanto antes. Avances en Supervisión Educativa, 18, 1-19. https://avances.adide.org/index.php/ase/article/view/531/371

Luengo, J. A. (2014). Cyberbullying. Prevenir y actuar. Hacia una ética de las relaciones en las redes sociales. Guía de recursos didácticos para centros educativos. Colegio Oficial de Psicólogos de Madrid.

Luengo, J. A. (2019). El acoso escolar y la convivencia en los centros educativos. Guía para el profesorado y las familias. Comunidad de Madrid.

https://www.comunidad.madrid/publicacion/ref/16443

Méndez, I., Ruiz Esteban, C., Martínez, J. P. y Cerezo, F. (2019). Ciberacoso según características sociodemográficas y académicas en estudiantes universitarios. Revista Española de Pedagogía, 77 (273), 261-276. https://doi.org/10.22550/REP77-2-2019-06

Nocito, G. (2017). Investigaciones sobre el acoso escolar en España: Implicaciones Psicoeducativas. Revista Española de Orientación y Psicopedagogía, 28(1), 104-118. https://doi.org/10.5944/reop.vol.28.num.1.2017.19361

Ortega-Ruiz, R, Del Rey, R. y Casas, J.A. (2016). Evaluar el bullying y cyberbulling validación española del EBIP-Q y del ECIP-Q. Psicología Educativa, 22(1), 71-79. https://doi.org/10.1016/i.pse.2016.01.004

Ortega-Ruiz, R. y Zych, I. (2016). La ciberconducta y la psicología educativa: retos y riesgos. Psicología Educativa, 22(1), 1-4. https://doi.org/10.1016/j.pse.2016.04.001

Perren, S., Corcoran, L., Cowie, H., Dehue, F., García, D., Mc Guckin, C. y Völlink, T. (2012). Tackling cyberbulling: Review of empirical evidence regarding successful responses by students, parents, and schools. International Journal of Conflict and Violence, 6, 283-293. https://doi.org/10.4119/UNIBI/iijcv.244

Pörhöla, M., Cvancara, K.; Kaal, E., Tampere, K. y Torres, B. (2016). Cross-cultural comparisons of bullying among university students: perspectives from Argentina, Estonia, Finland, and the United States. En H. Cowie y C.A. Myers (Eds.), Bullying among university students: cross-national perspectives (pp. 127-142). Routledge.

Prieto, M.T., Carrillo, J.C. y Lucio, L.A. (2015). Violencia virtual y acoso escolar entre estudiantes universitarios: el lado oscuro de las redes sociales. Innovación Educativa, 15(68), 33-47. https://www.redalyc.org/articulo.oa?id=179442126004

Redondo, J., Luzardo-Briceño, M., García-Lizarazo, K.L. y Inglés, C.J. (2017). Impacto psicológico del cyberbulling en estudiantes universitarios: un estudio exploratorio. Revista Colombiana de Ciencias Sociales, 8(2), 458-478 https://dx.doi.org/10.21501/22161201.2061

Romera, E.Mํ., Cano, J., García-Fernández, C. M.ạ. y Ortega-Ruiz, R. (2016). Cyberbulling: competencia social, motivación y relaciones entre iguales. Comunicar, 48(XXIV), 71-79. https://dx.doi.org/10.3916/C48-2016-07

Romero, A. y Plata, J.V. (2015). Acoso Escolar en las Universidades. Revista Enseñanza e Investigación en Psicología, 20(3), 266-274.

https://www.redalyc.org/articulo.oa?id=29242800003 
Salmivalli, C. (2010). Bullying and the peer group: a review. Agression and Violent Behavior, 15, 112-120. https://doi.org/10.1016/j.avb.2009.08.007

Sánchez Lacasa, C. y Cerezo, F. (2011) Estatus social de los sujetos implicados en bullying. Elementos para la mejora de la convivencia en el aula. Revista de orientación y psicopedagogía, 22(2), 137-149. https://doi.org/10.5944/reop.vol.22.num.2.2011.62

Smith, J.A. y Yoon, J. (2012). Cyberbulling presence, extent and forms in a midwestern postsecondary institution. Proceedings of the Information Systems Educator Conference, 11(3), 52-78.https://doi.org/10.1177\%2F2158244014526721

Tokunaga, R. S. (2010). Following you home from school: A critical review and synthesis of research on cyberbulling victimization. Computers in Human Behavior, 26, 277-287. https://doi.org/10.1016/i.chb.2009.11.014

Tovar, M.R., Fernández, M. y Fuentes, L. (2016). Acoso en la red: Cyberbulling. En M.C. PérezFuentes, J.J. Gázquez, Mํㅗ.M. Molero, A. Martos, A., M.․․ M. Simón, y A.B. Barragán (2016). La Convivencia Escolar: Un acercamiento multidisciplinar. Volumen II. ASUNIVEP, pp.19-24.

Tresgallo, E. (2020). Acoso escolar: los graves peligros de las redes sociales. Pautas de intervención. Pirámide.

Turan, N., Polat, O., Karapirli, M.; Uysal, C. y Turan, S.G. (2011). The new violence type of the era: Cyber bullying among university students: Violence among university students. Neurology, Psychiatry and Brain Research, 17(1), 21-26. https://doi.org/10.1016/ j.npbr.2011.02.005

Vergel, M., Martínez, J. y Zafra, S. (2016). Factores asociados al bullying en instituciones de educación superior. Revista Criminalidad, 58 (2), 197-208.

http://www.scielo.org.co/scielo.php?script=sci arttext\&pid=S1794-

$\underline{31082016000200008 \& \operatorname{lng}=e n \& n r m=i s o}$

Zych, I. Ortega-Ruiz, R. y Marín-López, I. (2016). Cyberbulling: a systematic review of research, its prevalence and assessment issues in Spanish studies. Psicología Educativa, 22(1), 5-18. https://doi.org/10.1016/j.pse.2016.03.0

Fecha de entrada: 5 diciembre 2019

Fecha de revisión: 6 noviembre 2020

Fecha de aceptación: 13 noviembre 2020 